\title{
Drei neue Synthesewege zur Herstellung von Polyolen für Polyurethane
}

\author{
Gerhard Behrendt, Martin Pohl, Peter Wagner, Hannelore Huth, Karl-Heinz Schmidt
}

\section{Einleitung}

Auf der Grundlage des Gesetzes zur Förderung der Kreislaufwirtschaft und Sicherung der umweltverträglichen Beseitigung von Abfällen (KrW-/AbfG)(1) fordert der Gesetzgeber eine zunehmende Verwertung von Abfällen und die auf Grund von Verordnungen oder freiwilligen Ruicknahmeverpflichtungen der Industrie existierenden Verwertungsquoten einzuhalten. Eine Verordnung, die für dieses Thema von Bedeutung ist, ist die Verordnung über die Vermeidung von Verpackungsabfällen (VerpackV) (2), eine weitere ist die freiwillige Rücknahmeverpflichtung der Industrie für Altkraftfahrzeuge.

Nach der VerpackV hat die Duales System Deutschland $\mathrm{GmbH}$ (DSD) als Garantiegeber zur Einhaltung der Verordnung fuir Kunststoffe aus der Sammlung eine Verwertungsquote von $72 \%$ garantiert. Das bedeutet, daß jährlich ca. 800.000 t Kunststoffe aus der Verpackung verwertet werden müssen. Da in zunehmendem Maße Kunststoffe eingesetzt werden, steigt diese Menge, insbesondere bei den Kunststoffflaschen. In diesem Kunststoffabfall sind alle Massenkunststoffe, dazu noch Verbundwerkstoffe und Gummi enthalten. Während die Polyolefine (Polypropylen und Polyethylen) durch Schwimm-Sink-Trennung weitestgehend abgetrennt werden können, gibt es derzeit für die restlichen Kunststoffe keine Verwertungsverfahren. In diesem Rest, jährlich ca. $135.000 \mathrm{t}$, sind jedoch wertvolle Materialien, vor allem Polyethylenterephthalat (PET) enthalten. Die PETMenge macht hier mit ca. 65.000 t/a auch die größte Menge aus.

Bei der Verwertung der Altautos ist die Wiederverwertung der Metalle unproblematisch und auch nicht Gegenstand der gegenwärtigen Diskussion. Problematisch ist die Shredderleichtfraktion, die bisher deponiert oder verbrannt wurde. Vor allem die Deponierung wird zukünftig kein Entsorgungsweg mehr sein können (3), so daß der Demontage der Kfz nach Ende ihrer Gebrauchsdauer größere Bedeutung zukommt. Ein Produkt, das volumenmäßig in den Altautos bedeutend ist, nämlich die Sitzkissen, stellt dabei für die Verwertung ein bisher nur unzureichend gelöstes Problem dar. Die Sitzkissen bestehen überwiegend aus Polyurethan- (PUR) Weichschaumstoff, für dessen Recycling es bisher zwar eine Reihe patentierter Verfahren gibt (4), von denen jedoch bisher keines angewendet wird. Ursache hierfuir ist einmal, daß aus dem Weichschaum bisher nur Polyole für PUR-Hartschaumstoffe minderer Qualität (Downcycling) hergestellt werden können und daß bei die- sen Verfahren cancerogene primäre aromatische Amine gebildet werden, die durch nachgeschaltete Verfahren zu ungefährlichen Produkten umgesetzt (5) oder abgetrennt werden müssen (6). Eine andere Variante besteht darin, mit einem Überschuß an einem Glykol den PUR-Weichschaum zu lösen und nur den Weichschaum-Polyetheralkohol herauszulösen, diesen abzutrennen, das Glykol im Kreislauf zu fahren und während des Kreislaufs durch Destillation aufzubereiten (7). Diese Verfahren werden aus rein ökonomischen Erwägungen heraus nicht genutzt, da die danach hergestellten Produkte deutlich höhere Herstellungskosten aufweisen als die Primärprodukte und darïber hinaus Weichschaumstoffe ergeben, die denen aus Primärprodukten bei weitem nicht gleichkommen.

\section{Eingesetzte Materialien und angewandte Methodik}

\subsection{Materialien}

Als Material wurden Lupranat M20A, Diethylenglykol, Dipropylenglykol und Polypropylenglykol 400 der BASF Schwarzheide GmbH, Polyethylenglykol 400 (Z4000) der Bayer AG, Di-n-butylamin und Triethylenglykol der Merck AG, Tris-1,3,5-(dimethylaminopropyl)hexahydro-1,3,5-triazin und Dibutylzinndilaurat der Nitroil AG sowie n-Hexylglycidether der Leuna-Chemie $\mathrm{GmbH}$ verwendet. Als Ausgangsprodukte der Abfallstoffe wurden drei Gruppen eingesetzt, von denen die Kfz-Sitzkissen von der Autoverwertung Graunke, Fürstenwalde, bzw. der Landgraf Kunststoffe, Fürstenwalde, zur Verfuigung gestellt und PET in Form verschiedener Sinkfraktionen der ENRETEC Polychemie Velten $\mathrm{GmbH}$ bzw. der Deutschen Gesellschaft für Kunststoff-Recycling mbH Köln, verwendet wurden. Die Zusammensetzung der Sinkfraktion wurde durch Dichtetrennung mit wässrigen Lösungen von Natriumchlorid bzw. Calciumchlorid ermittelt. Zur Abtrennung von reinem PET wurde ebenfalls die Dichtetrennung mit Calciumchlorid eingesetzt. Dazu wurde zunächst eine Lösung mit der Dichte von $1,2 \mathrm{~g} / \mathrm{cm}^{3}$ hergestellt und die Sinkfraktion zugegeben. Der aufschwimmende Anteil wurde abgetrennt. Der abgesunkene Teil wurde anschließend in eine Calciumchlorid-Lösung der Dichte 1,3 g/ $\mathrm{cm}^{3}$ gegeben. Der aufschwimmende Teil bestand $\mathrm{zu}$ uiber 99,5\% aus PET und wurde nach mehrmaliger Wäsche und Trocknung verwendet. Die abgesunkene Fraktion bestand im wesentlichen aus PVC und geringen Mengen hochgefuillten Polystyrens. 
Die Sitzkissen wurden von den Gestellen getrennt, die Bezuige entfernt und die Schaumstoffkissen entweder per Hand oder mittels einer Schneidmühle in ca. 30 - 40 $\mathrm{mm}$ große Flocken geschnitten. Weiterhin wurden Oligokondensate aus der PET-Produktion unterschiedlicher Zusammensetzung eingesetzt, die von der Ernst Michalke GmbH \& Co., Guben, zur Verfügung gestellt wurden. Die Oligokondensate bestehen im wesentlichen aus dem Dimeren Terephthalsäureester des Ethylenglykols mit der Strukturformel

$\mathrm{HO}-\mathrm{CH}_{2}-\mathrm{CH}_{2}-\mathrm{O}-\mathrm{CO} \bigcirc \mathrm{O}-\mathrm{CO}-\mathrm{O}-\mathrm{CH}_{2}-\mathrm{CH}_{2}-\mathrm{O}-\mathrm{CO}-O-\mathrm{CO}-\mathrm{O}-\mathrm{CH}_{2}-\mathrm{CH}_{2}-\mathrm{OH}$

zwischen 7 und 34 Gew.-\% Ethylenglykol, 3 bis 5 Gew.\% Diethylenglykol, etwa 2 Gew.\%-höheren Polyethylenglykolen und 0,5 bis 0,7 Gew.-\% Antimon-, Germaniumund Kobalt-Verbindungen (Veresterungskatalysatoren). Die Oligokondensate wurden in der originalen Zusammensetzung sowie nach Abdestillieren des Ethylenglykols im Vakuum-Rotations-Verdampfer $\left(165{ }^{\circ} \mathrm{C}, 20\right.$ mbar) bis auf 3 Gew.-\% verwendet; in der Ergebnisdiskussion werden sie folglich als Oligokondenat/original bzw. Oligokondensat/bearbeitet bezeichnet.

Die Schäumversuche wurden in Becherversuchen mit einem Rührgerät der Firma IKA mit stufenloser Drehzahlregelung und einem Tellerrührer sowie mit der Schäummaschine M20 der Firma Lackfa GmbH Chemnitz, durchgefuihrt.

\subsection{Methoden}

Die Solvolyse erfolgte in zwei unterschiedlichen Größenordnungen, einmal im 6 l-Sulfierkolben mit Rührer, Stickstoffeinleitung, Ruickflußkühler und Zugabetrichter, zum anderen im 90 l-Doppelmantel-Edelstahlreaktor mit externer Thermalölheizung mit Stickstoffeinleitung, Thermoelement, Zugabestutzen mit Trichter und Demister/Ruickflußkuihler. Die Rührgeschwindigkeit wurde in beiden Fällen auf $500 \mathrm{U} / \mathrm{min}$ eingestellt.

Generell wurden die Solvolysereagenzien im jeweiligen Reaktor in der weiter unten beschriebenen Menge vorgelegt und auf eine Lösungstemperatur erwärmt. Sobald die Lösungstemperatur erreicht war, wurde der Kunststoff zugegeben. Dabei wurde die Temperatur langsam gesteigert, anschließend wurde noch eine bestimmte Zeit bei einer vorgebenen Reaktionstemperatur geruihrt. Nach beendeter Reaktion wurde das Reaktionsgemisch abgekiihlt und bei einer Temperatur von ca. $100{ }^{\circ} \mathrm{C}$ durch ein $2 \mathrm{~mm}$-Sieb filtriert, um eventuell noch vorhandene Verunreinigungen abzutrennen.

\section{Ergebnisse}

\subsection{Glykolyse von PET}

PET wurde durch Umesterung an den Carbonsäureestergruppen der Terephthalsäure mit einem Überschuß an Glykolen unter Beteiligung der im Oligokondensat vorhandenen Glykole und Metallverbindungen als Umesterungskatalysatoren in Oligomere mit kürzeren Molekuilketten (niederen Molmassen) nach folgendem Schema umgesetzt:

$$
\begin{aligned}
& \text { PO- }-\mathrm{OH}+\mathrm{H}-\mathrm{O}-\mathrm{CH}_{2}-\mathrm{CH}_{2}-\mathrm{O}-\mathrm{CO} O-\mathrm{CO}-\mathrm{O}-\mathrm{CH}_{2}-\mathrm{CH}_{2}-\mathrm{OH} \\
& + \\
& \mathrm{H}-\mathrm{O}-\mathrm{CH}_{2}-\mathrm{CH}_{2}-\mathrm{O}-\mathrm{CO}-\mathrm{CO}-\mathrm{O}-\mathrm{R}-\mathrm{OH} \\
& \mathrm{R}+\mathrm{y}=\mathrm{n} \\
& \mathrm{CH}\left(\mathrm{CH}_{3}\right)-\mathrm{CO}-\mathrm{O}-\mathrm{CH}_{2}-\mathrm{CH}_{2}-\mathrm{OH}
\end{aligned}
$$

Während der Schmelzpunkt des PET zwischen 242 und $250{ }^{\circ} \mathrm{C}$ liegt, beträgt er für die Oligoesterkondensate $128^{\circ} \mathrm{C}$. Über die mittels DSC gemessenen Schmelzpunkte kann daher von einer weitgehenden Umesterung und Kettenspaltung ausgegangen werden.

Die Eigenschaften des durch die Umesterung PET hergestellten flüssigen Polyesterpolyols werden im wesentlichen durch zwei Parameter bestimmt: durch die Art und Menge des zur Umesterung verwendeten Glykols sowie durch die Reaktionsparameter Temperatur und Zeit. Daraus wird ersichtlich, daß es mit dem System aus PET, Oligokondensat und Glykol möglich ist, eine Vielzahl unterschiedlicher Produkte für ein

\begin{tabular}{|c|c|c|c|c|c|}
\hline Einsatzstoffe (in kg) & 0 & A & B & $\mathrm{C}$ & $\mathrm{D}$ \\
\hline Oligokondensat/original & 2,40 & 2,20 & 2,20 & 2,20 & 2,00 \\
\hline PET-Abfälle & 2,75 & 2,20 & 2,50 & 2,80 & 3,00 \\
\hline Diethylenglykol & - & 1,00 & 1,10 & 1,20 & 1,00 \\
\hline \multicolumn{6}{|l|}{ Reaktionsbedingungen } \\
\hline Reaktionstemperatur $\left({ }^{\circ} \mathrm{C}\right)$ & 228 & 220 & 220 & 220 & 220 \\
\hline Reaktionsdauer (Stunden) & 7 & 5 & 5 & 6 & 6 \\
\hline Hydroxylzahl (mg KOH/g) & 160 & 297 & 303 & 289 & 271 \\
\hline Viskosität (mPas, $20^{\circ} \mathrm{C}$ ) & 44.000 & 14.700 & 15.900 & 19.400 & 36.500 \\
\hline
\end{tabular}
breites Einsatzgebiet herzustellen. In der folgenden Tabelle sind einige Versuche dargestellt und die Produkte mit den für die zukünftige Verarbeitung wichtigsten Eigenschaften, dem Hydroxylgehalt (ausgedrückt als Hydroxylzahl oder kurz OH-Zahl) und der Viskosität: 
Eine weitere Versuchsreihe wurde mit Dipropylenglykol als alternativer Glykolkomponente und bearbeitetem Oligokondensat mit 3 Gew.-\% Monoethylenglykol durchgefuihrt:

\begin{tabular}{|c|c|c|c|c|}
\hline Einsatzstoffe (in kg) & $\mathrm{E}$ & $\mathrm{F}$ & G & $\mathrm{H}$ \\
\hline Oligokondensat/bearbeitet & 1,50 & 1,80 & 2,10 & 2,40 \\
\hline PET-Abfälle & 2,20 & 2,20 & 2,20 & 2,20 \\
\hline Dipropylenglykol & 1,30 & 1,30 & 1,30 & 1,30 \\
\hline \multicolumn{5}{|l|}{ Reaktionsbedingungen } \\
\hline Reaktionstemperatur $\left({ }^{\circ} \mathrm{C}\right)$ & 225 & 225 & 225 & 225 \\
\hline Reaktionsdauer (Stunden) & 6 & 6 & 6 & 6 \\
\hline Hydroxylzahl (mg KOH/g) & 267 & 263 & 258 & 255 \\
\hline Viskosität (mPas, $20^{\circ} \mathrm{C}$ ) & 10.500 & 11.200 & 10.800 & 11.700 \\
\hline
\end{tabular}

Weitere Versuche wurden mit anderen Verhältnissen der Einsatzkomponenten zueinander sowie mit weiteren Glykolen, hier vor allem Triethylenglykol und Polyethylenglykol 400 sowie Polypropylenglykol 400 durchgeführt. Einige Versuche dieser Reihen sind in der folgenden Tabelle zusammengestellt:

\begin{tabular}{l|lllll} 
Einsatzstoffe (in kg) & K & L & M & N \\
\hline $\begin{array}{l}\text { Oligokondensat/bearbeitet } \\
\text { PET-Abfälle }\end{array}$ & 1,80 & 1,80 & 1,80 & 1,80 \\
Dipropylenglykol & 2,20 & 2,20 & 2,20 & 2,20 \\
$\begin{array}{l}\text { Triethylenglykol } \\
\text { Polyethylenglykol 400 }\end{array}$ & - & - & - & - \\
Polypropylenglykol 400 & - & - & 1,80 & - & - \\
& - & - & - & 1,80 \\
Reaktionsbedingungen & & & & \\
Reaktionstemperatur $\left({ }^{\circ} \mathrm{C}\right.$ ) & 225 & 225 & 235 & 235 \\
Reaktionsdauer (Stunden) & 6 & 6 & 7 & 7 \\
\hline Hydroxylzahl (mg KOH/g) & 310 & 295 & 149 & 145 \\
Viskosität (mPas, 20 ${ }^{\circ} \mathrm{C}$ ) & 7.200 & 6.600 & 22.000 & 18.000
\end{tabular}

Die hier dargestellten Beispiele zeigen, daß es durch die Verwendung unterschiedlicher Glykole bei der Umesterung möglich ist, den Hydroxylgehalt und die Viskosität in einem großen Bereich zu variieren. Darüber hinaus ermöglicht das jeweils verwendete Glykol die Einstellung von speziellen Eigenschaften der daraus hergestellten Polyurethane (siehe Punkt 3.4) (8).

\subsection{Glykolyse von Polyurethanen}

Es wurde gefunden, daß sich die als Oligokondensate bezeichneten Ruickstände aus der Polyesterherstellung ebenfalls gut als Solvolysematerial für Polyurethane eignen. Bei Verwendung dieses Gemisches kann die Glykolyse durch die Möglichkeit der Anwendung höherer Temperaturen und die Verkürzung der Reaktionszeiten durch die in den Rückständen enthaltenen Katalysatoren günstiger als durch Anwendung der bisher uiblichen reinen Glykole durchgefuihrt werden, wobei die herge- stellten Polyole günstige Eigenschaften für die Herstellung von Polyurethan-Hartschaumstoffen aufweisen.

Durch dieses Verfahren werden Polyole für Polyurethane hergestellt, die durch Hydroxylzahlen im Bereich von 200 bis 650 und Viskositäten zwischen 6.000 und $200.000 \mathrm{mPas}\left(20^{\circ} \mathrm{C}\right)$ gekennzeichnet sind und aus den Reaktionsprodukten der Polyurethanabfälle mit dem bzw. den Glykolen sowie den Abfällen aus der Synthese von Polyestern erhalten werden und im wesentlichen aus dem den Polyurethanen zugrundeliegenden Polyolen, Oligoesteralkoholen, Glykolen sowie Aminoalkoholen und/oder Harnstoffen bestehen.

Die Umsetzung der Polyurethane verläuft formal analog der Umesterung der Carbonsäureester durch Umesterung an der Carbaminsäureestergruppe. R-NHCOO-R $+\mathrm{HO}-\mathrm{CH}_{2}-\mathrm{CH}_{2}-\mathrm{OH} \rightarrow \mathrm{R}-\mathrm{NHCOO}-\mathrm{CH}_{2}-\mathrm{CH}_{2}-$
$\mathrm{OH}+\mathrm{R}^{\prime}-\mathrm{OH}$

Dabei bedeuten $\mathrm{R}$ den Rest des aromatischen Di- oder Polyisocyanats und R' den Rest des Polyetheralkohols, meistens eines Triols, das auf der Grundlage von Glycerol oder Trimethylolpropan durch anionische Polymerisation mit Ethylenoxid und/oder Propylenoxid hergestellt wird.

Zusätzlich dazu wirken jedoch Glykole als nucleophiles Reagens auf die in den Weichschaumstoffen vorliegenden Polyharnstoffe, die aus der Treibreaktion mit Wasser und den Isocyanatgruppen gebildet werden. Da das Verhältnis von Hydroxylgruppen aus Wasser und aus Polyol bei den Formulierungen für Weichschaumstoffe üblicherweise im Bereich von 3:1 liegt, ist der Anteil der Harnstoffgruppen größer als der der Urethangruppen. Die Harnstoffgruppen werden durch die Glykole vor allem zu primären aromatischen Aminen abgebaut:

R-NHCO-NH-R + HO- $\mathrm{CH}_{2}-\mathrm{CH}_{2}-\mathrm{OH} \rightarrow \mathrm{R}-\mathrm{NHCO}-\mathrm{O}-\mathrm{CH}_{2}$ $\mathrm{CH}_{2}-\mathrm{OH}+\mathrm{R}-\mathrm{NH}_{2}$

Zur Entfernung des bei der Umsetzung der PolyurethanWeichschaumstoffe entstehenden primären aromatischen Amins ist seine Abtrennung oder Umsetzung mit leicht reagierenden Gruppen erforderlich. Nach einer Vorschrift der BASF AG (5) wird diese „Entaminierung“ durch Reaktion von Glycidethern mit den primären Aminogruppen vorgenommen; dadurch entstehen Aminoalkohole, die bei der Umsetzung mit Polyisocyanaten katalytisch wirken, so daß ihre Menge im Polyol sorgfältig kontrolliert werden muß. Die Reaktion läßt sich formal folgendermaßen darstellen:

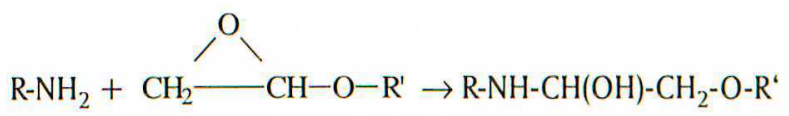

Folglich ist der Reaktionsablauf bei diesen Versuchen insoweit modifiziert, daß während der Umsetzung der Glycidether in mehreren Anteilen zugesetzt wird (5). Beispielhaft für viele Ansätze seien folgende Beispiele angefuihrt: 


\begin{tabular}{|c|c|c|c|c|}
\hline Einsatzstoffe (in kg) & Q & $\mathrm{R}$ & S & $\mathrm{T}$ \\
\hline Oligokondensat/original & 25,0 & 1,25 & 25,0 & 25,0 \\
\hline PUR-Weichschaum-Abfälle & 50,0 & 3,5 & 50,0 & 50,0 \\
\hline n-Hexylglycidether & 0,8 & 0,2 & 1,5 & 2,75 \\
\hline \multicolumn{5}{|l|}{ Reaktionsbedingungen } \\
\hline Reaktionstemperatur $\left({ }^{\circ} \mathrm{C}\right)$ & 175 & 165 & 165 & 165 \\
\hline Reaktionsdauer (Stunden) & 8 & 6 & 7 & 5,5 \\
\hline Hydroxylzahl (mg KOH/g) & 368 & 386 & 374 & 368 \\
\hline Viskosität (mPas, $20^{\circ} \mathrm{C}$ ) & 17.200 & 15.000 & 12.400 & 14.360 \\
\hline
\end{tabular}

Der Vorteil der Umsetzung der Polyurethane mit den Oligokondensaten liegt darin, daß die Anwendung deutlich niedrigerer Temperaturen bei ansonsten gleicher Reaktionsdauer möglich wird. Weiterhin ist eine zusätzliche Katalyse in der Regel nicht erforderlich. Durch die niedrigeren Temperaturen bedingt, ist die Menge an primären aromatischen Aminen im Reaktionsgemisch deutlich geringer als in den Fällen, in denen bei höherer Temperatur und mit Glykolen gearbeitet wird, so daß auch die Menge an Glycidether verringert werden konnte.

\subsection{Kombinierte Glykolyse/Aminolyse von Polyurethanen}

Abgeleitet aus Arbeiten zur Analyse von Polyurethanen (9) zur Bestimmung von Nebengruppen, die aus anderen Reaktionen der Isocyanate resultieren, wurde die Spaltung der Urethangruppe in einem Gemisch aus Glykolen und sekundären aliphatischen Aminen untersucht. Bei dieser Umsetzung reagiert die Urethangruppe vorwiegend mit dem sekundären Amin nach folgendem Reaktionsschema:

$\mathrm{R}-\mathrm{NH}-\mathrm{COOR}$ ' $+\mathrm{HNR}_{2} \rightarrow \mathrm{R}-\mathrm{NH}-\mathrm{CO}-\mathrm{NR}_{2}{ }_{2}+\mathrm{R}$ - $-\mathrm{OH}$

Das Reaktionsmedium ist durch die sekundären Amine basisch, so daß die nucleophile Spaltung der Harnstoffe deutlich reduziert wird und deren Menge unterhalb 1 Gew. $\%$ liegt. Die Glykole werden dabei so ausgewählt, daß einmal die Reaktivität ihrer Endgruppen etwa in der Größenordnung der freigesetzten Polyetheralkohole liegt und zum anderen die durch sie verringerte Kettenlänge zwischen den Vernetzungspunkten (mittlere Molmasse zwischen den Vernetzungen) nicht in einen Bereich abfällt, durch den Polyurethane mit unbrauchbaren Eigenschaften entstehen. Als Glykole wurden deshalb vorzugsweise längerkettige eingesetzt, wie die folgenden Beispiele zeigen:

\begin{tabular}{l|llll} 
Einsatzstoffe (in kg) & W & X & Y & Z \\
\hline $\begin{array}{l}\text { PUR-Weichschaum-Abfälle } \\
\text { Di-n-butylamin }\end{array}$ & 3,20 & 3,20 & 3,20 & 3,20 \\
$\begin{array}{l}\text { Dipropylenglykol } \\
\text { Triethylenglykol }\end{array}$ & 0,060 & 0,060 & 0,060 & 0,060 \\
Polyethylenglykol 400 & 0,60 & & & \\
Polypropyle & & 0,60 & & \\
& & & 0,90 &
\end{tabular}

\begin{tabular}{l|llll} 
Reaktionsbedingungen & & & & \\
Reaktionstemperatur $\left({ }^{\circ} \mathrm{C}\right)$ & 200 & 200 & 200 & 200 \\
Reaktionsdauer (Stunden) & 3 & 4 & 5 & 5 \\
\hline Hydroxylzahl (mg KOH/g) & 310 & 307 & 295 & 289 \\
Viskosität (mPas, $20^{\circ} \mathrm{C}$ ) & 1.830 & 2.360 & 4.540 & 4.180
\end{tabular}

Die Reaktionsprodukte sind klare Flüssigkeiten (wenn einheitliche Schaumstoffe verwendet werden) mit vergleichsweise niedriger Viskosität und hoher katalytischer Aktivität (10). In einem Falle wurde bei Verwendung von Polyurethan-Weichschaumstoff auf der Basis von Toluylendiisocyanat ein kristalliner Bodensatz beobachtet.

\subsection{Polyurethane aus den Polyolen}

Die Polyole werden mit dem Ziel hergestellt, daraus wieder hochwertige Polyurethane zu produzieren. Dazu ist es erforderlich, die Polyole in auf die Polyole zugeschnittenen Systemen zu formulieren. Solche Formulierungen wurden für alle hier beschriebenen Polyole entwickelt und erprobt.

Das Hauptanwendungsgebiet der PET-Polyole liegt auf dem Hartschaumstoffsektor. Schaumstoffe erfordern den Einsatz von Treibmitteln. Auf physikalische Treibmittel wurde bei der Entwicklung der Formulierungen verzichtet, da diese in der Regel nur zu weiteren Problemen führen; auf den Einsatz von Chlorfluorkohlenwasserstoffen (FCKW) wird generell verzichtet, die Ersatzstoffe Pentan oder Cyclopentan erfordern explosionsgeschuitzte Anlagen, die Anwendung von flüssigem Kohlendioxid bedingt einen neuartigen Maschinentyp zur Verarbeitung. Es wurde aus diesen Gründen auf die bekannte Reaktion des Wassers mit den Isocyanatgruppen unter Bildung von Harnstoffen und Kohlendioxid zurïckgegriffen:

$$
2 \mathrm{R}^{\prime}-\mathrm{NCO}+\mathrm{H}_{2} \mathrm{O} \rightarrow \mathrm{R}^{\prime}-\mathrm{NH}-\mathrm{CO}-\mathrm{NH}-\mathrm{R}^{\prime}+\mathrm{CO}_{2} \uparrow
$$

Die bei dieser Reaktion entstehenden Polyharnstoffe haben mehrere Vorteile: sie stabilisieren die Polymerkette, erhöhen den Stickstoffgehalt (was zur Flammfestigkeit beiträgt) und führen zu höheren Reaktionstemperaturen im Gemisch und damit zu schnellerer Aushärtung. Nachteilig ist die Tendenz der Schaumstoffe, bei übermäßig hohem Anteil an Harnstoffgruppen zu verspröden.

Durch die Auswahl spezieller Katalysatoren, die üblicherweise für die Herstellung von PUR-Hartschaumstoffen nicht, dafuir aber bei der Herstellung von Isocyanuratschaumstoffen eingesetzt werden, gelang die Herstellung von PUR-Hartschaumstoffen mit ïberraschend guten Eigenschaften bei ausschließlicher Verwendung der Recyclat-Polyole. Die Hartschaumstoffe werden mit einem Unterschuß an Polyisocyanat hergestellt, wobei eine Formulierung typischerweise ist: 
Polyol

$93,5 \mathrm{~g}$

Wasser

$2,4 \mathrm{~g}$

Tris-1,3,5-(dimethyl-aminopropyl)-hexahydro-1,3,5-triazin 2,2 Lupranat M20A $70,0 \mathrm{~g}$

Der Isocyanatindex beträgt in der Regel 75, folgende Reaktionszeiten werden eingestellt:

$\begin{array}{ll}\text { Rührzeit } & 10 \mathrm{~s} \\ \text { Startzeit } & 17 \mathrm{~s}\end{array}$

Je nach Hydroxylzahl kann die Menge des Polyols in den Rezepturen schwanken. Dadurch verändern sich auch die weiteren Reaktionszeiten Steigzeit und Klebfreizeit sowie die Eigenschaften, insbesondere die Dichte der Hartschaumstoffe. Eine Zugabe von Stabilisatoren ist in der Regel nicht erforderlich.

Einige Beispiele für Hartschaumstoffe aus verschiedenen der oben beschriebenen Polyole enthält die folgende Zusammenstellung (11):

\begin{tabular}{l|llll} 
Polyol & $\mathrm{G}$ & $\mathrm{L}$ & $\mathrm{N}$ & $\mathrm{S}$ \\
& $64,5 \mathrm{~g}$ & 73,7 & 114,7 & 44,5 \\
Wasser & $2,1 \mathrm{~g}$ & 2,1 & 3,0 & 2,0 \\
Tris-1,3,5-(dimethyl-aminopropyl) & & & & \\
-hexalyydro-1,3,5-triazin & $2,0 \mathrm{~g}$ & 2,0 & 1,2 & 0,5 \\
Lupranat M20A & $70,0 \mathrm{~g}$ & 70,0 & 70,0 & 70,0
\end{tabular}

Die Reaktionszeiten und Eigenschaften der Hartschaumstoffe:

\section{Steigzeit (s)}

Klebfreizeit (s)

Rohdichte $\left(\mathrm{g} / \mathrm{dm}^{3}\right)$

Offenzelligkeit (\%)

Druckfestigkeit (MPa)

Dimensionsstabilität $\left(24 \mathrm{~h} / 140^{\circ} \mathrm{C}\right)$

$\begin{array}{llll}47 & 51 & 62 & 41 \\ 62 & 65 & 86 & 45 \\ 34 & 35,5 & 35 & 30 \\ 11 & 13 & 9 & 7 \\ 0,063 & 0,059 & 0,069 & 0,056 \\ +1,5 \% & +2,0 \% & +5 \% & +1,7 \%\end{array}$

Aus den Polyolen W bis Z können ebenfalls Hartschaumstoffe hergestellt werden, jedoch war das Ziel dieser Arbeiten die Herstellung elastischer Produkte. Es lassen sich aus diesen Recyclat-Polyolen ohne Zusatz von Primärpolyolen erstmalig elastische Polyurethane als Gießelastomere und als halbharte Schaumstoffe, z. B. fuir Schockabsorber, herstellen (12).

\section{Diskussion}

Durch die drei an der Technischen Fachhochschule Wildau neu entwickelte Verfahren zum Recycling der Kondensationspolymere Polyethylenterephthalat und Polyurethan-Weich-Schaumstoffe lassen sich Abfälle davon in neue, zur Herstellung von Polyurethanen mit guten Eigenschaften geeignete Polyole nach einfachen Verfahren gewinnen. Für die technische Anwendung dieser Verfahren ist wesentlich, daß einmal ausreichend Kunststoff-Abfälle zur Verfügung stehen, die Verfahren technisch einfach beherrschbar sind, immer zur gleichen Qualität der Produkte führen, die Produkte auf dem Markt mit Gewinn absetzbar sind. Nach bisherigem
Kenntnisstand stehen sowohl PET-Abfälle als auch die Oligokondensate in so großen Mengen zur Verfügung, daß bei Ausschöpfung aller Quellen daraus ca. 200.000 t/a Polyol hergestellt werden könnten, was wesentlich mehr als der gegenwärtige Bedarf in Westeuropa ist. Bei den Polyurethan-Weichschaumstoffen liegt das Aufkommen, bedingt durch die gegenwärtige Situation auf dem Entsorgungsmarkt und die Dumpingpreise vieler Deponiebetreiber, bei wesentlich geringeren Mengen. Nach Greifen der TASi im Jahre 2005 wird sich die Situation jedoch umkehren, da dann die Shredderabfälle nicht mehr deponiert werden dürfen.

Die hier vorgestellten Verfahren sind einfach zu beherrschende Umsetzungen, die in vergleichsweise einfachen Anlagen durchgefuihrt werden können. Die Maßstabsvergrößerung vom jetzigen $100 \mathrm{l}$-Maßstab in den 0,5 $\mathrm{m}^{3}$-Maßstab oder darüber hinaus wird derzeit vorbereitet.

Die Untersuchungen in der Technikumsanlage der Technischen Fachhochschule Wildau haben gezeigt, daß sich die Polyole auch bei Schwankungen in der Zusammensetzung aller Eingangsstoffe mit einer Qualität innerhalb enger Toleranzen herstellen lassen. Die Schwankungen innerhalb der Toleranzen werden zusätzlich durch die dazu speziell entwickelten Formulierungen abgefangen, so daß letztendlich Polyurethane gleicher Eigenschaften hergestellt werden.

Ein wesentliches Kriterium sind die Herstellungskosten im Vergleich zu den auf dem Markt befindlichen Primärprodukten. Umfangreiche Berechnungen haben gezeigt, daß bei den PET-Polyolen der break-even-point bei einer Jahresmenge von $200 \mathrm{t}$ erreicht wird, daß bei einer Jahresproduktion von $1000 \mathrm{t}$ ein deutlicher Überschuß erwirtschaftet werden kann. Bei den Polyolen aus PURWeichschaum ist der break-even-point wesentlich von dem angewandten Verfahren abhängig. Bei der Umsetzung mit nachgeschalteter Deaminierung wird dieser Punkt erst bei über 1000 t/a erreicht, bei der kombinierten Aminiolyse/Glykolyse dagegen bei etwa 250 t/a.

\section{Ausblick}

Auf Grund der guten Eigenschaften der Recyclat-Polyole, der einfachen und sicheren Verfahren ohne Entstehung neuer Abfälle sowie der günstigen Herstellungskosten haben mehrere Unternehmen des Landes Brandenburg Interesse an der Übernahme der Verfahren bekundet. Für zwei der angemeldeten Schutzrechte $(11,12)$ wurden inzwischen Lizenzen vergeben. In einem Unternehmen wird gegenwärtig eine Versuchsanlage zur Herstellung von Polyolen aus Weichschaumstoffen betrieben; in einem weiteren Unternehmen läuft gegenwärtig der Bau einer Pilotanlage für die Herstellung von Polyesterpolyolen, mit der in der ersten Ausbaustufe $500 \mathrm{t} /$ a hergestellt werden sollen. Zwei weitere mittelständische Unternehmen werden die dort hergestellten Produkte in Form von Systemen einsetzen und damit bisher verwendete Primärprodukte ablösen. 
Die Arbeiten zum weiteren Ausbau der hier dargestellten Synthesewege mit anderen Kunststoffen und mit anderer Zielrichtung werden systematisch fortgesetzt. Dabei stehen grundlagenwissenschaftliche Untersuchungen des Reaktionsablaufs und die Erweiterung der Einsatzmöglichkeiten im Mittelpunkt der Arbeiten.

\section{Danksagung}

Wir danken der Bundesstiftung Umwelt, dem Bundesministerium für Bildung, Forschung und Wissenschaft sowie der Technologie- und Innovationsagentur Brandenburg für die finanzielle Unterstiitzung der Arbeiten sowie den genannten Unternehmen BASF AG, Bayer AG, Hoechst Trevira GmbH, Nitroil AG und Th. Goldschmidt AG für die Bereitstellung der Reaktanden. Dank gilt auch den Unternehmen der Entsorgungsbranche, ganz besonders der ENRETEC Polychemie Velten, und der EPIK GmbH Fürstenwalde sowie der Graunke Autoverwertung Fürstenwalde für die Bereitstellung und Aufbereitung der Kunststoff-Abfälle.

\section{Literatur}

[1] BGBI. I, S. 2705 vom 27.9.1994

[2] BGBl. I, S. 1234 vom 12.6.1991

[3] A. Oberholz, Umweltmagazin 4/1999, S. 18

[4] siehe z. B. G. Bauer, FAT-Schriftenreihe Nr. 86, Verfahren zur Umwandlung polymerer Mischabfälle aus der Autositzproduktion in Polyole; G. Bauer in: G. Menges, W. Michaeli, M. Bittner, Recycling von Kunststoffen, CarlHanser-Verlag, München-Wien 1992, S. 265-274; E. Grigat, Hydrolyse von Polyurethanen, Kunststoffe $\underline{68}$ (1978), 284, F. Simioni, S. Bisello, Polyol Recovery from Rigid Polyurethane Waste, Cellular Polymers $\underline{2}$ (1983), 281; USP 2.937.151, USP 3.117.940; USP 4.115.298

[5] B. Naber, V. Neiß, M. Gassan, Polyurethane 1995, Preprints, S. 287-290; Gassan, Naber, Neiss, Möckel, Weißpflog, DE-OS 4234 335; EP 0592 952;

[6] W. Raßhofer, T. Münzmann, Chemolyse, speziell Hydrolyse von Polyurethanen, in W. Raßhofer, Recycling von Polyurethanen, Carl-Hanser-Verlag, München, 1995, S. 237 A. Demharter „Glykolyse von Polyurethanreststoffen aus dem Bau- und Industriebereich“, in W. Raßhofer, Recycling von Polyurethanen, Carl-Hanser-Verlag, München, 1995, S. 379 - 384; USP 4.035.314; USP 4.044.046; (6) DEOS 251 663; DE-OS 2557 172; DE-PS 2738572

[7] USP 4.159.972

[8] HAVEL-Chemie AG; Erfinder: G. Behrendt, A. Lobeda, M. Pohl, Verfahren zur Herstellung von Polyesteralkoholen sowie Polyesteralkohole, DE-Anmeldung (1998); G. Behrendt, A. Lobeda, M. Pohl, Verfahren zur Herstellung von Polyolen aus Polyurethanabfällen und durch dieses Verfahren erhältliche Polyole, DE-OS 19719084 (1997)

[9] G. Behrendt, Über den Einfluß von Isocyanuraten auf Struktur und Eigenschaften von Urethan-Polymeren, Berlin, 1972
[10] G. Behrendt, M. Pohl, Verfahren zur Herstellung von Polyolen und diese Polyole, DE-OS 19817536

[11] G. Behrendt, M. Pohl, Verfahren zur Herstellung von Polyurethanen, DE-OS 19817539

[12] G. Behrendt, M. Pohl, Polyurethanmassen und Verfahren zu ihrer Herstellung, DE-OS 19852151

\section{Autoren}

Prof. Dr. rer. nat. Gerhard Behrendt,

Dipl.-Chem. Martin Pohl,

Dr. rer. nat. Peter Wagner,

Dipl.-Chem. Hannelore Huth,

Dipl.-Ing. Karl-Heinz Schmidt

Technische Fachhochschule Wildau,

FB Ingenieurwesen/Wirtschaftsingenieurwesen

Friedrich-Engels-Straße 63

15745 Wildau

Tel. (0 33 75) 508591 